\title{
Computational study of hole shape effect on film cooling performance
}

\author{
Jun Yao ${ }^{1,2}$ and Yufeng Yao $^{2 *}$ \\ ${ }^{1}$ Mitsubishi Electric R\&D Centre Europe B.V., 20 Frederick Sanger Road, The Surrey \\ Research Park, Guildford, Surrey, GU2 7YD, UK \\ ${ }^{2}$ School of Aerospace and Aircraft Engineering, Faculty of Engineering, Kingston \\ University, 104 Friars Avenue, Roehampton Vale, London SW15 3DW, UK
}

The manuscript was received on प and was accepted after revision for publication on $\square$.

DOI: $10.1177 / 0957650911399013$

\begin{abstract}
Film-cooling effectiveness has been studied by using a computational approach based on solving the Reynolds-averaged Navier-Stokes equations. A wind tunnel test configuration is considered with a total of four cooling hole geometries as a cylindrical hole, a cylindrical hole with an upstream wedge (called 'ramp' thereafter), a shaped diffuser, and a double console slot. In all cases, the hole centre-line has an inclination angle of $35^{\circ}$ against the mainstream airflow and the blowing ratio is unity. Choosing the cylindrical model as a baseline, simulations have been carried out for grid convergence and turbulence model influence studies. Results are compared with available experimental data and other numerical predictions and good agreement has been achieved. Further computations continue with three remaining geometries, using the baseline flow conditions and configuration. Comparing to the results from the baseline model, it was found that the centre-line adiabatic cooling effectiveness has shown incremental increases for the 'ramp' model, while results from the console slot model and the shape diffuser model have exhibited significant improvements by a factor by 1.5 and 2, respectively. The reason for such a step change in cooling effectiveness is mainly due to the weakening of the vortex structures in the vicinity of the hole exit, thus significantly reducing the entrainment of surrounding 'hot' fluids.
\end{abstract}

\section{Keywords: 매, nin}

\section{INTRODUCTION}

The HP turbine blades operate under 'hot' environmental conditions, whereby the efficiency is often limited by the maximum temperature under which the blade materials can endure. This can be further related to the life of blade, i.e. the number of cycles it undergoes while in service. As a result, turbine blades have to be routinely checked and replaced when their maximum cycles are reached. To reduce the operating cost, blade designers have been researching the method to extend the blade lifetime. By injecting a coolant airflow at various locations of blade surface is obvious a simple and very efficient way and this

${ }^{*}$ Corresponding author: School of Aerospace and Aircraft Engineering, Faculty of Engineering, Kingston University, 104 Friars Avenue, Roehampton Vale, London SW15 3DW, UK. email:y.yao@kingston.ac.uk technique (normally called 'blade cooling') has been widely used by turbo-machinery industry.

The blade film=cooling technique is particularly used for the mid-span portion of a blade and the aim is to introduce a 'cooled' thin-layer fluid film on the blade surface, thus preventing it from direct exposure to a 'hot' gas mainstream from a combustor. The coolant airflow is taken from the engine compressor and goes into a reservoir where it has been accelerated through an internal cooling duct camber inside the blade and finally exited out of the blade surface through array of cooling holes. While the surface temperature is kept lower, blades can be used for a longer period of time before being replaced due to deformation or degradation, and therefore the cost of maintenance and replacement can be largely reduced.

To implement this technique, blade surface will have a large number of holes that are arranged in 
several rows across the spanwise direction at locations where the blade surface is largely exposed to a high-temperature gas stream. Typical locations include blade leading edge area (for leading-edge impinging cooling), mid-blade surface region (for blade film cooling) and blade trailing edge area (for trailing-edge injection cooling). This study merely focuses on the blade film cooling. Some previous studies [1-4] have shown that the jet exit hole shape plays an important role in determining the adiabatic cooling effectiveness that is often used as a quantitative measure of how blade surface could be protected. In addition, the exit hole shape will also have influences on the cooling envelopes/boundaries, such as the cooling distance in the longitudinal direction and the cooling spreading in the lateral direction. Due to a 'lifted-off' effect of counter-rotating vortex pair (CRVP) in the near field of a hole exit, the 'cooled' jet flow tends to be 'separated or detached' from the blade surface and this will cause the significant reduction of the cooling effectiveness. Other factors such as blowing ratio and operating conditions could also have certain effects on the cooling effectiveness.

The traditional film cooling configuration uses a cylindrical tube that links an internal cooling duct to the external cross flow domain. The jet hole shape becomes an elliptic type at the exit and its size (and the longitudinal axis length) is subject to an inclination angle (which is normally around 30$35^{\circ}$ ). With the advancement in manufacture technology, it is now possible to produce other more sophisticated hole geometries, both internally and externally, to allow the blade designers to optimize their design in order to maximize the cooling effectiveness. In the light of this, the present study is to carry out a detailed computational fluid dynamics (CFD) analysis on some representative modern film cooling configurations in order to determine the most suitable hole shape with better cooling performance for the next generation of blade cooling hole design and assessment.

The study starts with a comprehensive analysis using a base cylindrical model, previously studied by other researchers [5] and validated against available numerical predictions and experimental measurement. Using this model as a baseline, further three hole geometries are considered as a cylindrical hole with an upstream wedge geometry (named 'ramp' thereafter) [6], a shaped diffuser hole geometry [7], and a double console slot geometry [8]. The results analysis will focus on the evaluation of adiabatic cooling effectiveness, temperature distributions, and flow and vortex structures in the near and far wake fields.

\section{PROBLEM DESCRIPTION}

The configurations of the base and the ramp models follow the studies of Kapadia et al. [5] and Jiang et al. [6]. The shaped diffuser model is a variant of that used by Dhungel [7] and the console slot model adopts the settings as seen in reference [8]. A suite of general CAE simulation software ANSYS-CFX [9] is used for the solid modelling, the meshing, and the CFD computations.

\subsection{Geometry definition}

The dimensions of mainstream cross flow domain, the cooling tube, and the plenum domain are all based on the tube diameter ' $D$ '. This will ensure that the scalability of the problem, and given the same inflow conditions and the diameter ' $D$ ', the simulation Reynolds number will remain the same and thus results are comparable.

The computational domain is $49 D \times 3 D \times 10 D$ for the cross flow domain and $22 D \times 3 D \times 4 D$ for the coolant plenum, in the streamwise, the spanwise, and the wall normal directions, respectively (Fig. 1), where $D$ is diameter of the base (cylindrical hole) model, i.e. $D=2.54 \mathrm{~mm}$. The coordinate origin ' $\mathrm{O}$ ' is located at the trailing-edge point of the hole exit, resulting in a distance of $20 \mathrm{D}$ from inlet plane to the trailing edge of the hole exit and $29 \mathrm{D}$ from the trailing edge of the hole exit to the outlet plane of the cross flow domain in the streamwise direction. The plenum domain has smaller lengths of $22 D$ in the streamwise direction. Both the cross flow and the plenum domains have the same spanwise lengths of $3 D$ (note that for the shape diffuser and console slot models, the spanwise length needs to be extended in order to accommodate larger hole exit. Detailed definition is given in below). The plenum is located at a distance of $2 D$ below the bottom wall surface of the mainstream domain.

For the base (cylindrical) model, the inclination angle of cooling hole to the mainstream flow is $\alpha=35^{\circ}$ (Fig. 2) and the angle has been kept the same for all test cases. For the external ramp model (Fig. 3), the cylindrical tube and the coordinate origin remains the same and a wedge with a length of $2 D$ and a height of $h=0.5 \mathrm{D}$ is located at $2.75 \mathrm{D}$ upstream of the origin ' $\mathrm{O}$ '. In reality, an additional 'wedge' or a cluster of 'wedge array' on the blade surface, despite of its small size, will affect the aerodynamic behaviours of the blade and sometime may adversely degrade the blade efficiency. At the same time, however, it may also be possible that the 'wedge' could play a role similar as a 'vortex generator', thus it may 


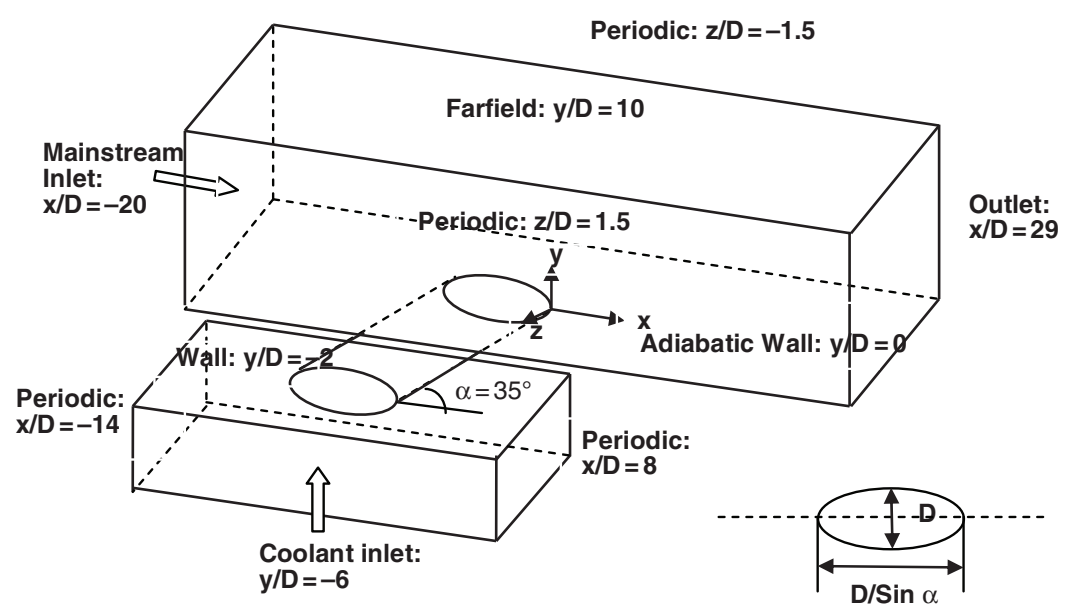

Fig. 1 A sketch of computational domain with the baseline 'cylindrical' model showing the tube cooling hole arrangement

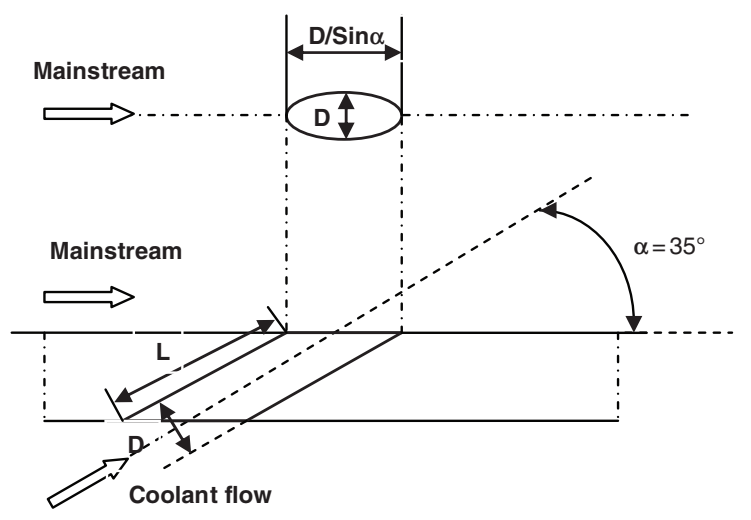

Fig. 2 A sketch of the baseline 'cylindrical' model with an inclination angle of $35^{\circ}$

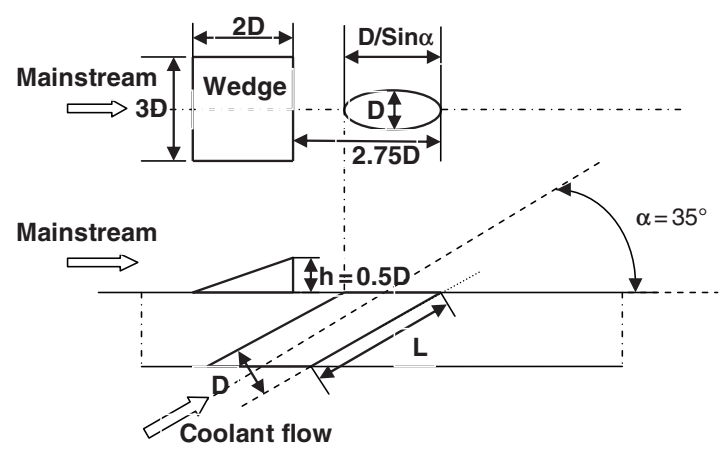

Fig. 3 A sketch of the 'ramp' model with an external 'wedge' shape upstream of the jet exit

even be able to improve the blade performance at the near stall point. Nevertheless, a full assessment of its impact in blade performance needs to be carried out before adopting this concept.

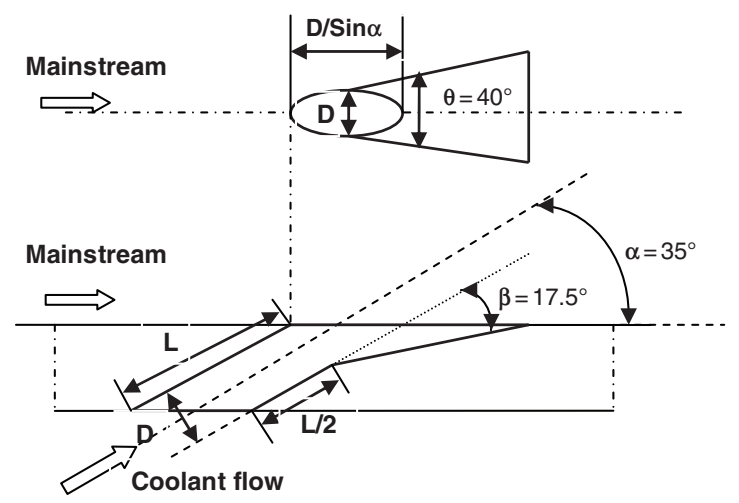

Fig. 4 A sketch of the 'shape diffuser' model with a deflected hole trailing edge line at the middle of cooling hole

The shaped diffuser model has the same cylindrical tube shape linking to the plenum domain and a diffuser-type shape starts half a way of the tube length and links to the cross flow domain (Fig. 4). While the leading edge line of the cooling hole remains unchanged, the trailing-edge line deflected at an angle of $17.5^{\circ}$ after a distance of about $3 D$ from hole inlet and on the bottom wall surface of the mainstream domain (i.e. $y / D=0$ ), the lateral expansion angle between two opposite hole edge lines is $\theta=40^{\circ}$. A previous study by Dhungel [7] used a deflection angle of $\beta=15^{\circ}$ (resulting in $30^{\circ}$ inclination angle of the jet centre-line). In order to keep the same inclination angle as that in the base model case, the deflection angle for the present shaped diffuser model has been increased to $\beta=17.5^{\circ}$, resulting in a $35^{\circ}$ inclination angle. As this change will lead to a 
wider hole exit width of about $3 D$, thus the spanwise length of the cross flow domain has been increased to $9 D$.

The console slot model geometry (Fig. 5) follows the same dimensions of a previous wind tunnel experimental study [8]. While the domain arrangement is similar to that of the base model, the tube diameter $D=25 \mathrm{~mm}$ is now large about a factor of 10 compared to the base model (thus Reynolds number based on this length scale would be ten times higher). Thereby lengths of other dimensions are increased by the same factor and in particular, a length of $200 \mathrm{~mm}$ is used in the spanwise to accommodate the jet exit slot. Other parameters are the exit throat slot height $S=5 \mathrm{~mm}$ and the exit slot width (or console hole pitch) $W=50 \mathrm{~mm}$ (i.e. the whole slot width is $100 \mathrm{~mm}$ ). This configuration was primarily designed for a wind tunnel test study (Sargison [8]), and if this cooling hole concept being used on a real turbine blade, the diameter has to be reduced accordingly.

\subsection{Mesh generation}

The hybrid mesh topologies (with structured meshes in the near wall region and unstructured meshes in the far field) are applied using advanced meshing

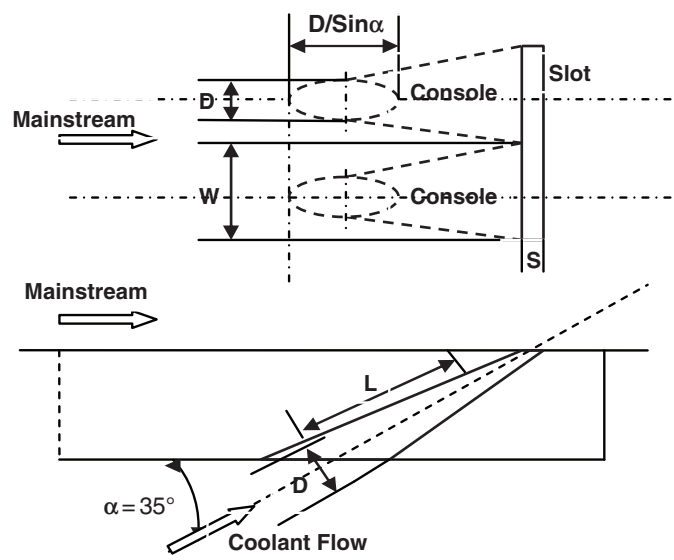

Fig. 5 A sketch of the 'Console Slot' cooling hole model. Note the hole diameter is $25 \mathrm{~mm}$ larger than other models tools in the ANSYS software. In order to reserve periodicity for the spanwise direction in the cross flow domain and the plenum domain, two periodic pairs were defined to ensure exactly periodicity of mesh points.

Some special care has been taken for the near wall regions (particularly in the vicinity of jet hole exit), where an inflation viscous layer is generated to resolve the boundary layer and the thermal layer. For the fine grid, about 10-15 inflation layers with an expansion factor ranging from 1.2 to 1.4 are used with the first layer height of about $0.02 \mathrm{~mm}$, resulting in a wall grid size in the wall unit of $y^{+} \approx 6-7$, which is suitable for the $k-\varepsilon$ turbulence model and its variants (e.g. the re-normalized group (RNG) $k-\varepsilon$ model). The coarse and the medium grids are also generated using slightly less restrictive meshing parameters in the near wall region, resulting about 50 per cent and 75 per cent less elements respectively, in comparison to that from fine grid. For the console slot model, similar meshing criteria are applied, considering a factor of ten in real physical dimensions. Table 1 gives the summary of grid refinements, grid resolutions, and total number of elements for each model.

\subsection{Boundary conditions}

For the cross flow domain, a fixed uniform velocity of $104 \mathrm{~m} / \mathrm{s}$ and a total temperature of $300 \mathrm{~K}$ are given at the inlet plane. These conditions are taken from previous wind tunnel tests [10] and numerical simulations [5]. At the outlet plane, zero-gradient conditions are applied. At top surface, a free-slip wall condition is used and a non-slip adiabatic wall condition for the ground wall surface. The periodic condition is also applied on the periodic pair in the spanwise direction. For the coolant tube wall and the ceiling of the plenum domain, the non-slip adiabatic wall conditions are defined. Similar to that in cross flow domain, periodic conditions are used for the periodic pair in the plenum domain in the spanwise direction, while a slip wall condition is used for two side walls in the streamwise.

The velocity boundary condition at the plenum inlet has to be carefully defined such that a unity

Table 1 Summary of grid refinement, grid resolution, and mesh statistics

\begin{tabular}{lll}
\hline Models & Average $\Delta y^{+}$ & Number of elements $\left(\times 10^{6}\right)$ \\
\hline Baseline (coarse grid) & 40 (duct), 14.22 (wall) & 1.35 \\
Baseline (medium grid) & 40 (duct), 10.04 (wall) & 1.85 \\
Baseline (fine grid) & 40 (duct), 6.66 (wall) & 2.50 \\
Ramp & 41.2 (duct), 5.91 (wall) & 1.64 \\
Shape diffuser & 36.5 (duct), 6.31 (wall) & 2.25 \\
Console slot & 166 (duct), 5.34 (wall) & 3.57 \\
\hline
\end{tabular}


blowing ratio of the cross flow inlet momentum and the 'mean' jet flow exit momentum (i.e. $\left.m=\rho_{\mathrm{j}} u_{\mathrm{j}} / \rho_{\mathrm{cf}} u_{\mathrm{cf}}\right)$ is maintained as close as possible (ideally around 99.5 per cent in accuracy). Since this ratio depends on both the density and velocity ratios of the cross flow and the coolant jet, given the fixed cross flow momentum and the temperature of $150 \mathrm{~K}$ at the plenum inlet in the simulation, the ideal averaged jet exit velocity should be about a half of the cross flow velocity, i.e. $52 \mathrm{~m} / \mathrm{s}$. In each simulation, an initial estimated velocity, based on potential flow mass conservation at the plenum inlet and the jet exit plane is derived and in case of base model, this gives an initial guess of a plenum inlet velocity of $0.619 \mathrm{~m} / \mathrm{s}$. Due to viscous effect in the simulation, the predicted blowing ratio generally cannot retain as a unity and based on the simulation results, some adjustments of the plenum inlet velocity value is required by tuning the final blowing ratio of unity. This results in a coolant jet velocity of about $0.471 \mathrm{~m} / \mathrm{s}$ for the baseline cylindrical model, $0.6188 \mathrm{~m} / \mathrm{s}$ for the ramp model, and $0.796 \mathrm{~m} / \mathrm{s}$ for the shape diffuser model. For the console slot model, initial guess of the plenum inlet velocity is $0.217 \mathrm{~m} / \mathrm{s}$ (again based on the mass conservation of the console slot hole exit and the plenum inlet). It is worth noting that flow and thermal conditions used in this study were taken from other relevant experimental wind tunnel studies. For turbine rig testing and actual operation conditions, the inlet temperature of the cross flow domain will be much higher around $1500-2000 \mathrm{~K}$ and also the coolant jet flow extracting from the compressor stage will be high around $500-700 \mathrm{~K}$. So, the temperature ratio could be around a factor of 3-4. This will affect the coolant jet velocity if a unity blowing ratio needs to be achieved.

\subsection{Adiabatic cooling effectiveness}

The quantitative results of adiabatic cooling effectiveness is defined as

$$
\eta=\left(T_{\mathrm{aw}}-T_{\mathrm{cf}}\right) /\left(T_{\mathrm{j}}-T_{\mathrm{cf}}\right)
$$

where the $T_{\mathrm{aw}}$ is the computed adiabatic wall temperature, $T_{\text {cf }}$ the cross flow inlet total temperature (300 K), and $T_{\mathrm{j}}$ the plenum inlet temperature (150 K).

\subsection{Grid convergence study}

The grid refinement study was carried out using the base cylindrical model on a coarse grid, a medium grid, and a fine grid (Table 1). The difference of these three meshes is mainly due to different grid resolution requirements for the bottom wall surface of the mainstream domain, while other meshing parameters are kept the same. The RNG $k-\varepsilon$ turbulence model is used in the simulations (note that the turbulence model influence will be studied and described later). The coolant jet velocity of $0.471 \mathrm{~m} / \mathrm{s}$ is used for the fine grid case and this value needs to be tuned slightly (less than 3 per cent) for the coarse and the medium grids. Figure 6 gives a comparison of the adiabatic cooling effectiveness along the centre-line of the jet exit from trailing edge point up to the outlet. The results show that there is little difference between the coarse and the medium grids results in the near wake region of the coolant jet exit (up to $x / D=7$ ), while in downstream after $x / D=10$, small differences appear. Comparing to experimental measurement by Sinha et al. [10], results from the fine grid have shown better agreement, particularly, in the near wake region close to the coolant jet exit $(x / D<5)$. The reason for this is probably due to the improved near wall grid resolutions with at least one grid point inside the sublayer $\left(y^{+} \leq 10\right)$ is used (Table 1$)$. In order to have better grid resolution for capturing flow and thermal structures, it was decided to use the fine grid for the remaining simulations.

\subsection{Turbulence model effect}

The simulation Reynolds number (Re) is estimated about 17000 based on free-stream velocity $(104 \mathrm{~m} / \mathrm{s})$ and hole diameter $(D=2.54 \mathrm{~mm})$ for the base model, the ramp model and the shape diffuser model, and about 170000 for the console model (due to a larger hole inlet diameter $D=25 \mathrm{~mm}$ ). The flow will be turbulent and for computation based on the Reynoldsaveraged Navier-Stokes (RANS) approach, suitable turbulence model is required. The initial turbulence model selection studies were carried out using the base (cylindrical) model, by comparing the results

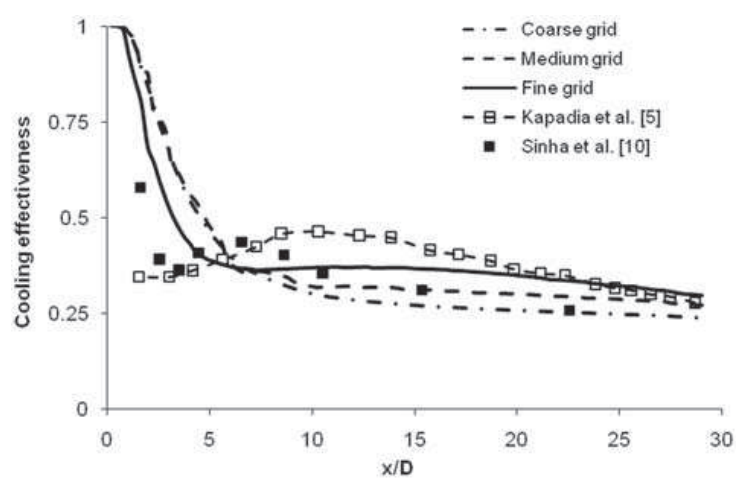

Fig. 6 Comparison of centre-line cooling effectiveness from coarse, medium, and fine grids of base model with other computations and test data 


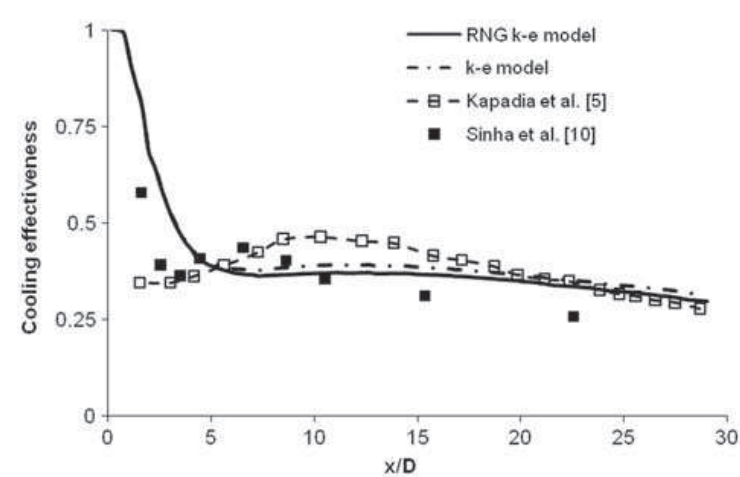

Fig. 7 Comparison of centre-line cooling effectiveness from RNG $k-\varepsilon$ and standard $k-\varepsilon$ turbulence models of base model with other computations and test data

from the standard $k-\varepsilon$ model and its variant the RNG $k-\varepsilon$ model. It was found that there is no significant difference in terms of the centre=line adiabatic cooling effectiveness (Fig. 7) and the flow structures (not shown here). As simulation time using the standard $k-\varepsilon$ model takes about as twice as much compared to that by the RNG $k-\varepsilon$ model, it was decided to use the RNG $k-\varepsilon$ model for all simulations.

All simulations start with a uniform initial flow field and run until a steady state converged status with a convergence residual of $10^{-5}$. In general, this can be achieved by $75-150$ iterations and it takes about 150-240 min on a dual-core P4 desktop computer.

\section{RESULTS AND DISCUSSIONS}

\subsection{Validation study of the base (cylindrical) model}

The base cylindrical model has been used for both the grid refinement study (as described above) and the plenum inlet velocity 'trial and error' tests. By using the estimated plenum inlet velocity of $0.619 \mathrm{~m} / \mathrm{s}$, for a coarse grid with larger $y^{+}$at the near wall regions, simulated cooling effectiveness has shown considerable discrepancies (about a factor of 2 lower than the experimental data). After progressive mesh refinement to the fine grid, results have been improved and lie close to the test data in further downstream, but still considerably lower in the near field of the hole exit as seen in Fig. 8. To understand the cause of this discrepancy, some visualizations of flow field have been carried out and it was found that the flow characteristics of the recirculation inside the tube near the entrance and the mixing with mainstream flow and the development of the CRVP are qualitatively similar to that obtained by other researchers [5]. However,

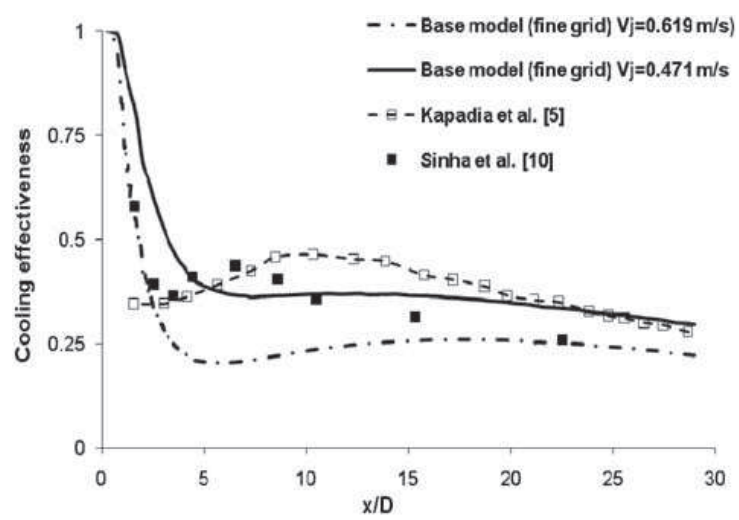

Fig. 8 Comparison of centre-line cooling effectiveness from base model with other computations and test data

the penetration depth of the coolant jet flow seems overpredicted, and this results in the film being liftedoff from the surface wall. 'Hot' gas stream has been entrained towards the near wall region, causing low cooling effectiveness.

Based on the fine grid study, it is clear that the reason for the low cooling effectiveness is due to some incorrect considerations in the physical model set-up. While the 'cooled' jet fluid meets with the 'hot' cross flow fluid, there will be a thermal mixing process. Along this line, further simulations have been performed, whereby the forced mixing process of two fluids mixing was tested. However, results did not lead to the overall improvement of the cooling effectiveness predictions (not shown here).

After careful examination of all predefined conditions, it was noted that the initial guess of plenum inlet velocity actually leads to the overprediction of mass flowrate at the jet hole exit, thus a unity blowing ratio was in fact not reserved. Hence, this inlet velocity needs to be manually tuned, based on the difference of a predicted blowing ratio at the jet hole exit and the target unity blowing ratio. After some 'trial and error' tests, the plenum inlet velocity is finally set to be $0.471 \mathrm{~m} / \mathrm{s}$ in order to meet a unity blowing ratio.

Figure 8 shows the comparison of centre-line adiabatic cooling effectiveness in the streamwise direction. Using the finally tuned plenum inlet velocity of $0.471 \mathrm{~m} / \mathrm{s}$, RANS predictions are in good agreement with that of Kapadia et al. [5] using more advanced detached-eddy simulation (DES) approach for $x /$ $D>6$, and both results overpredict the cooling effectiveness compared to the measurement by Sinha et al. [10]. In the near field close to hole exit $(x / D=0-6)$, present RANS simulation has given the right trend, but overpredicts at worst by a factor of 2, compared to the test data, while DES result has shown good 


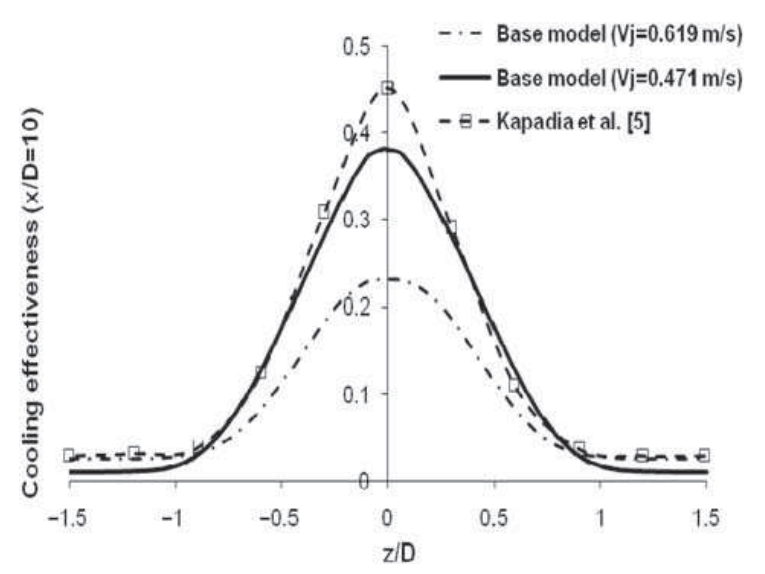

Fig. 9 Comparison of spanwise cooling effectiveness at $x=10 D$

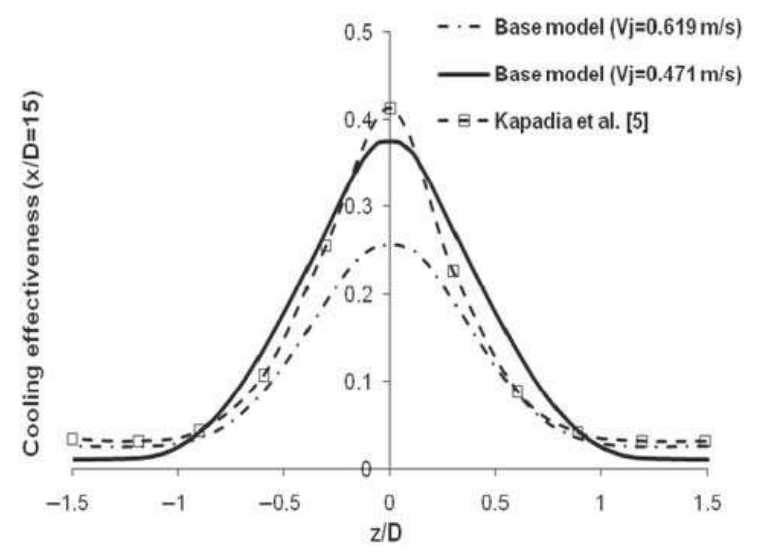

Fig. 10 Comparison of spanwise cooling effectiveness at $x=15 D$

comparison up to $x / D=2.5$. Further analyses are made at two successive cross-planes at $x / D=10,15$ (Figs 9, 10), showing that good comparisons with reference data [5] are achieved over whole spanwise length.

Similar to that observed in reference [5], flow recirculation near the cooling tube entrance is clearly visible by the velocity vector plot in Fig. 11 . This is due to the sharp edge between the plenum ceiling and the coolant tube. As large flow separation will lead to significant blockage inside the tube, it is desirable to avoid or minimize this, probably by smoothing at the local corner area, e.g. elliptical circumference, to ensure a less sudden change in the flow direction, as discussed in reference [7].

\subsection{Evaluation of cooling effectiveness}

Simulations continue with three additional variants of hole geometry shapes, namely the ramp model, the shaped diffuser model, and the console slot model. In each case, the same flow conditions as those used for the base model are retained, apart from the plenum inlet that needs to be tuned in order to meet a unity blowing ratio, such that a fair comparison on the cooling effectiveness enhancement by varying the cooling hole geometry can be made. As the coordinate origin at the hole exit trailing edge is different in shape diffuser model case, all comparisons will be made at same downstream distance referencing to the origin located the exit hole trailing edge position.

Figures 12-14 depict the comparisons of the computed cooling effectiveness from four models with the experimental measurement of Sinha et al. [10] for the base model, Dhungel for the shaped diffuser model [7] and Sargison [8] for the console slot model. Note that for these three cases, there is unfortunately lack of measurement data in the jet exit region $x / D=0-1.5$ that is important to evaluate the cooling efficiency of various configurations.

In general, the centre-line cooling effectiveness from all four cases follows the same trend. Results of the base model and the ramp model have shown a rapid reduction of cooling effectiveness till $x / D=6$; after that a slow decrease happens. In contrary to a similar study by Jiang et al. [6], our simulation does not shown any centre=line cooling enhancement by an upstream wedge geometry with $h=0.5 D$, and in the vicinity of the hole exit, the cooling effectiveness is similar to that of base model, while some improvements are observed in lateral direction due to the deflection effect of the wedge. Overall, the console slot model and the shaped diffuser model have shown improved cooling effectiveness in the near wake region up to $x / D=15$. Note that the results from shaped diffuser have been averaged over the spanwise in order to compare with those of Dhungel [7], otherwise it would have much higher local centre=line cooling effective, about a factor of 2 compared to that of the base model. Figures 13 and 14 give the cooling effectiveness distributions in the spanwise direction. Comparing to the base model, the cooling effectiveness from the ramp model prediction has almost same peak value at the centre-line (i.e. agrees with that observed in Fig. 12), but has slower decay in the lateral direction while away from the central area. This indicates that on average, the overall cooling effectiveness of the ramp model would be better than that of the base cylindrical model. Further visualization of local flow field (see Fig. 17, to be discussed later) confirms that this is mainly owed to the effect of the wedge geometry that will deflect the 'hot' incoming fluid and slightly weaken the CRVP magnitude, hence allow the coolant jet flow to remain in the near wall 

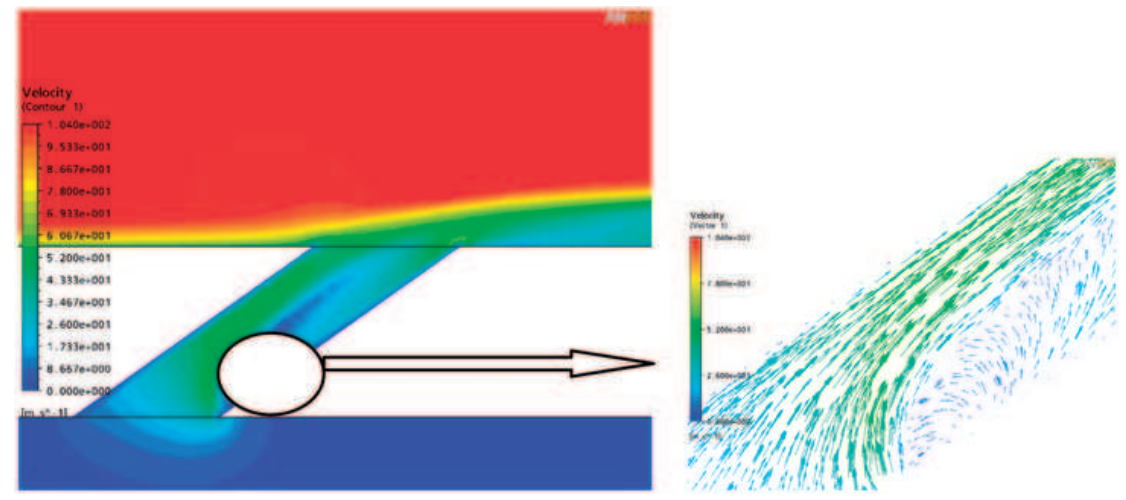

Fig. 11 Velocity contours at a plane across the jet centre=line and velocity vectors near inside cooling pipe showing recirculation flow

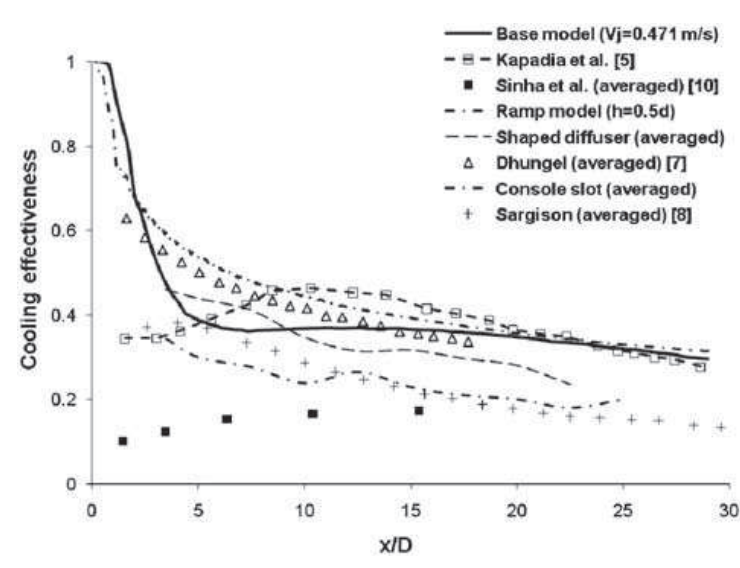

Fig. 12 Centre=line cooling effectiveness. (Note: results of shaped diffuser model and Dhungel data are averaged in spanwise direction.)

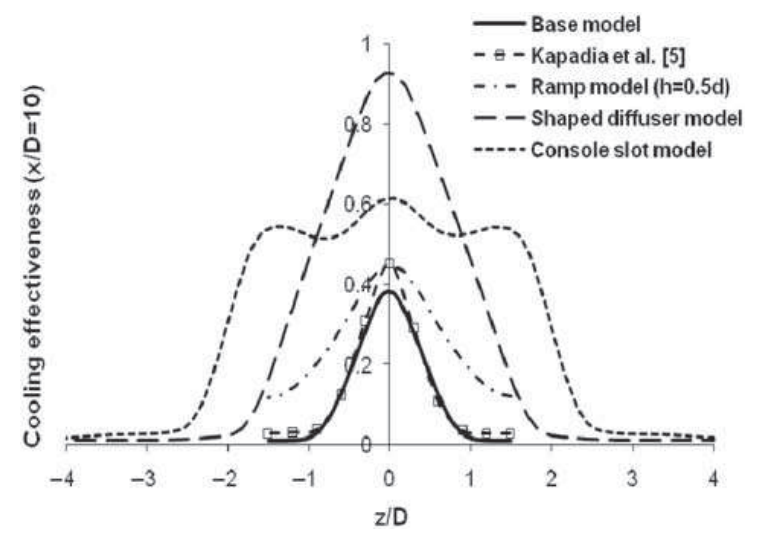

Fig. 13 Comparison of spanwise cooling effectiveness at $x=10 D$

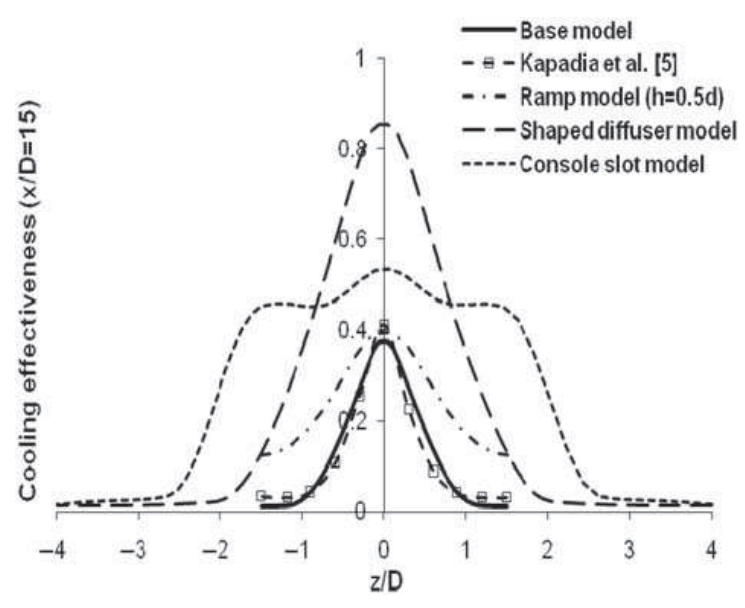

Fig. 14 Comparison of spanwise cooling effectiveness at $x=15 D$

within extended distances from the jet hole exit. Comparing to the base model and ramp model, results of the shaped diffuser model and the console slot model did demonstrate much better cooling effectiveness across the spanwise with higher peaks at centre-line, about a factor of 1.5 and 2 from the console model and the shape diffuser model, respectively. The reasons are partially due to large jet hole exit area that will reduce the coolant jet exit velocity, thus largely reducing the jet penetration and the 'cooled' gas could retain in the near wall region. Other factors such as the strength of CRVP will be discussed later.

\subsection{Temperature distributions}

The temperature distributions on the bottom wall surface of the mainstream domain and the middle 

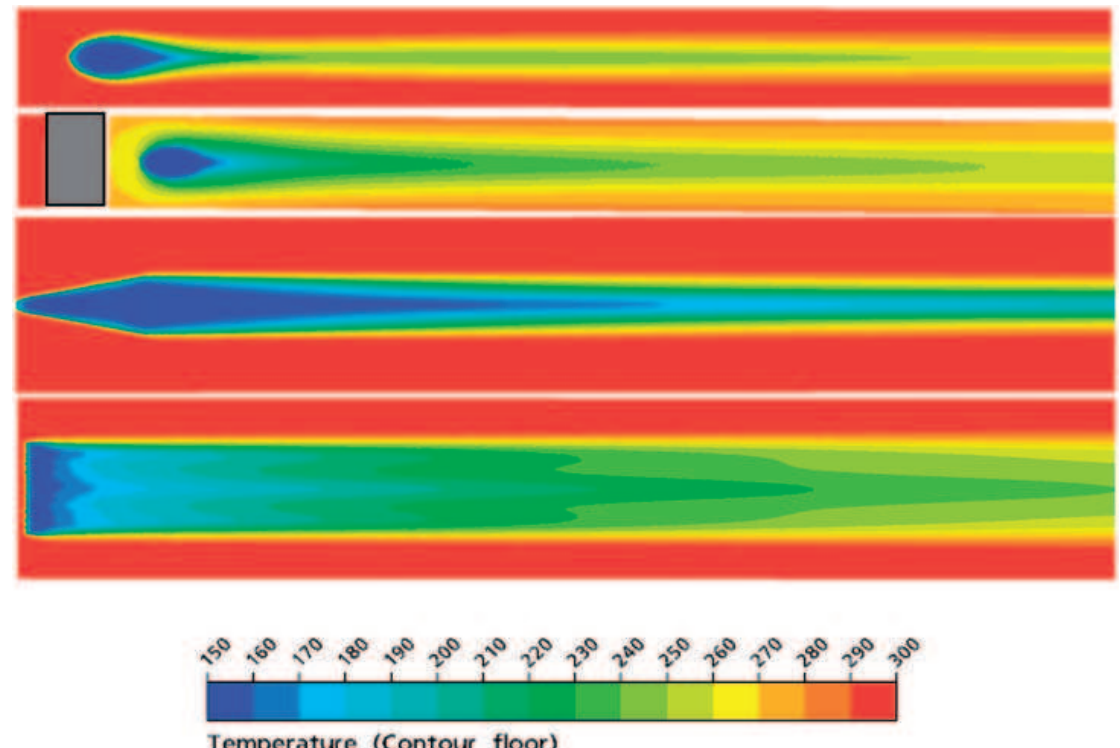

Fig. 15 Temperature contours on the bottom wall surface of the mainstream domain in an order of (from top to bottom) base model, ramp model, shaped diffuser model, and console slot model. The contour levels are also shown

plane of the coolant jet hole are illustrated in Figs 15 and 16, which show the influence of the coolant jet in the vicinity of the jet hole exit and downstream in the wake region. It can be seen that the cooled thin-layer film lasts before it is 'lifted-off' from the wall surface due to the flow mixing and entrainment of the 'hot' cross flow fluids. Simulation shows that the base model has smallest cooling coverage area, comparing to other three models. By introducing a small wedge upstream of the hole exit, the ramp model does show significant improvement of cooling effectiveness in lateral direction, mainly due to the effect of deflecting the 'hot' mainstream fluid. However, as previously discussed, its impact on blade design aerodynamic characteristics and stage efficiency need to be carefully considered and assessed before adopting this concept in the blade cooling design. Both the shape diffuser and the console slot concepts show significant cooling effectiveness improvement. While the console geometry covers a wider area due to an effect known as the 'Coanda' effect as discussed by Sargison [8], which means that the cooled film remains attached to the surface due to the nature of the console duct shape, the shape diffuser model exhibits prolonged cooling coverage in the longitudinal direction (Fig. 16). This is due to the deflection of trailing edge portion of the cooling hole that slows down the coolant flow speed and causes the coolant fluid almost attaching to the wall along the way up to the hole exit and beyond.

\subsection{Flow and thermal characteristics}

Figure 17 displays velocity vectors on a cross plane through the centre-line of the cooling hole. In case of the console slot model, there is a pair of cooling holes in a side-by-side arrangement. The vector graph was initially produced on a plane through each cooling hole centre for comparison. As they are almost identical, only one graph is used here. For the base (cylindrical) model, Fig. 17(a) illustrates the flow features, similar to those previously observed by Kapadia et al. [5]. In the ramp model, due to the existence of a wedge geometry, flow recirculation exists in the wedge trailing edge region (Fig. 17(b)) and furthermore the 'hot' mainstream fluid has been deflected away from the wall surface. While this may help in keeping the low-speed cooling flow direction unaffected from the high-speed mainstream flow after the hole exit, the penetration depth of coolant fluid jet into the mainstream flow seems broadly unchanged in comparison to the base model case. The hole expansion in the shape diffuser model slows down the flow very significantly in a region near the hole exit (Fig. 17(c)). In this study, a deflection angle of $17.5^{\circ}$ was applied and any further increase of this angle will increase the risk of causing local reverse flow, thus leading to the potential dangerous situation of sucking the 'hot' mainstream fluid into the cooling hole. Results from the console slot model reveal very thin film layer in comparison to 

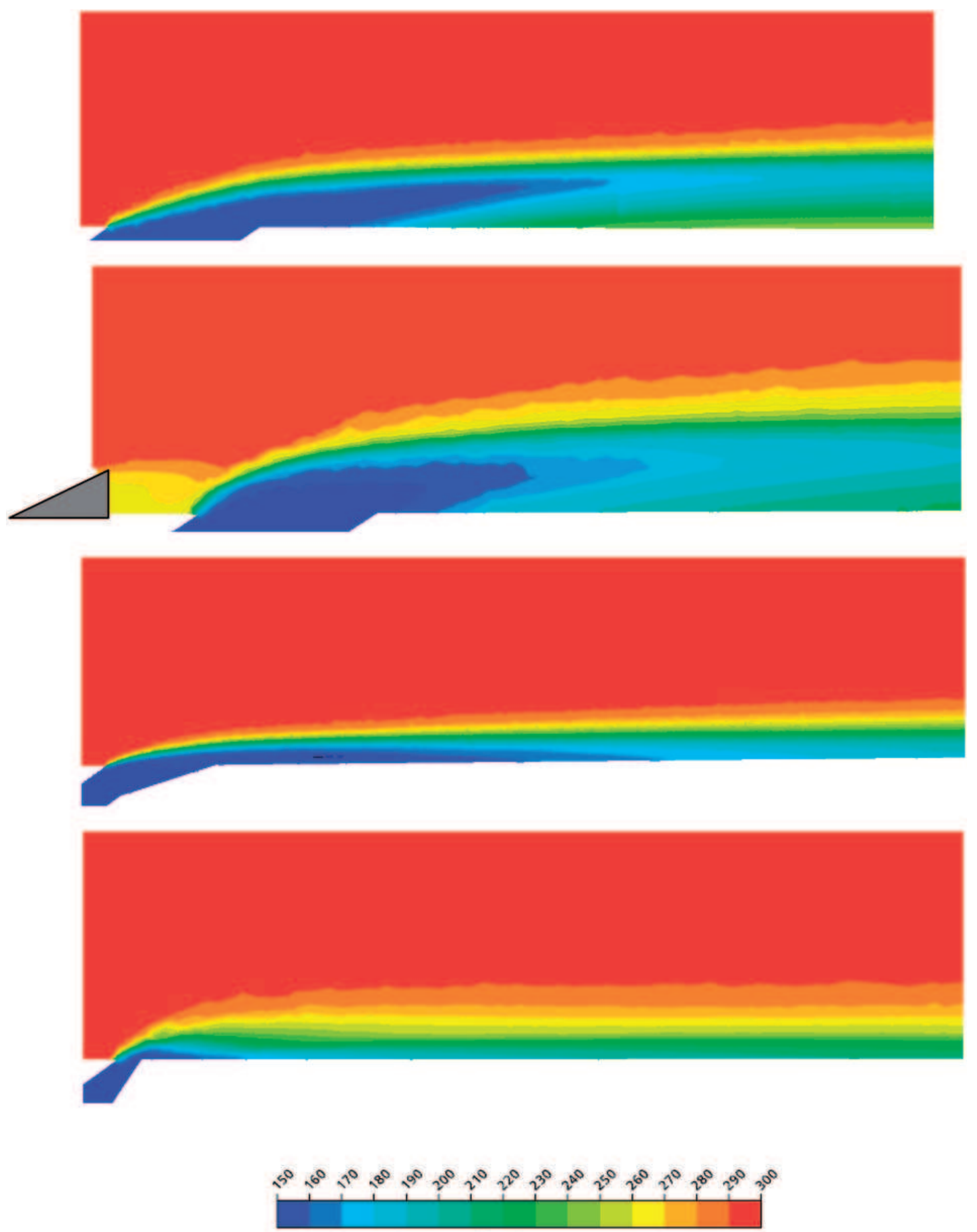

Temperature (Contour floor)

Fig. 16 Temperature contours on a middle plane of cooling hole of the mainstream and coolant domains in an order of (from top to bottom) base model, ramp model, shaped diffuser model, and console slot model. The contour levels are also shown 

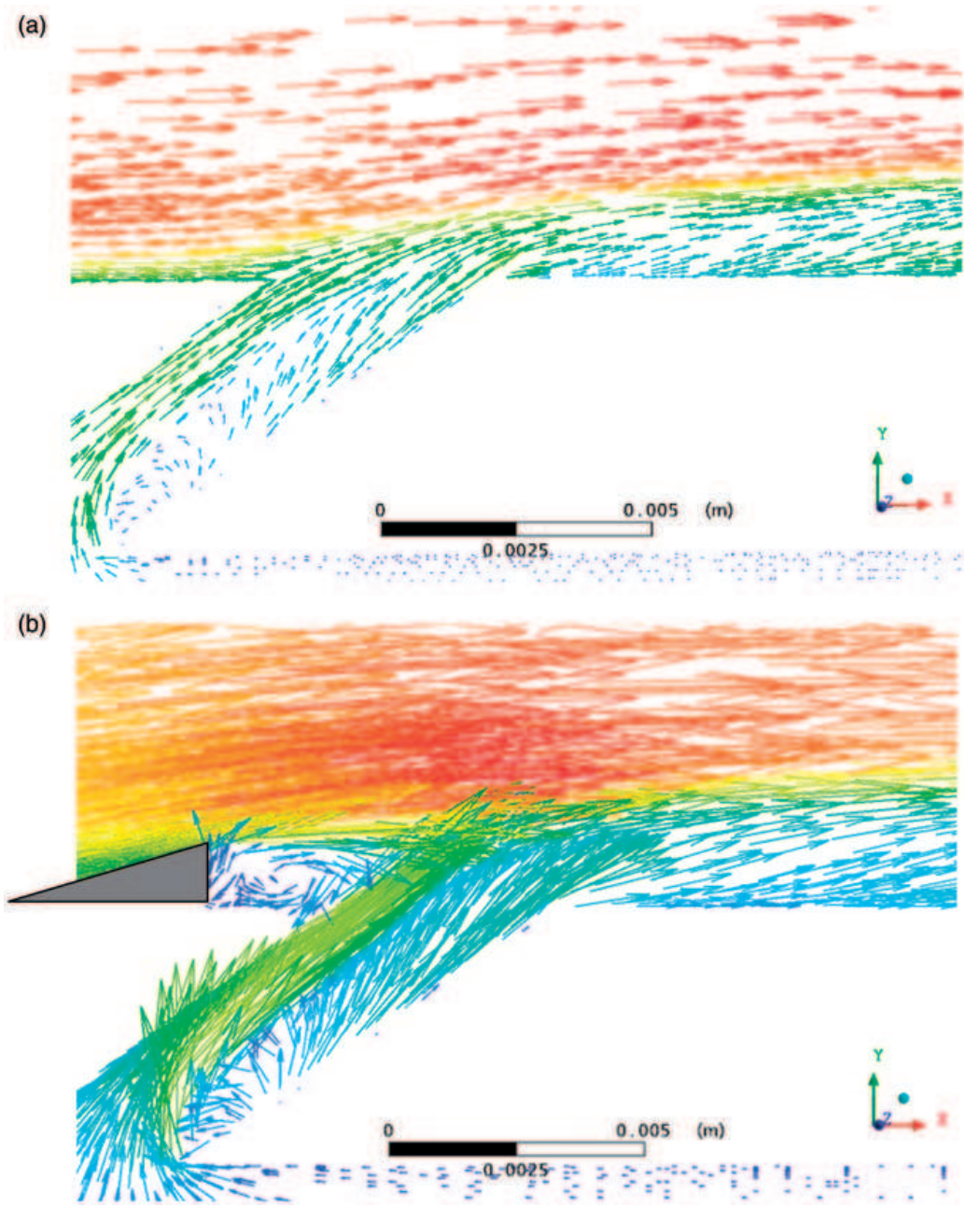

Fig. 17 Velocity vectors on a middle plane of cooling hole of the mainstream and coolant domains: (a) base model, (b) ramp model, (c) shaped diffuser model, and (d) console slot model

other three models, despite its advantages of a wider coverage area in the lateral direction (Fig. 15). Figure 18 exhibits temperature contours at eight successive cross planes $(x / D=0,1,3,6,10,15,20$, and 25) from the hole exit up to the domain outlet plane. Temperature contours on the bottom wall surface of the mainstream domain (Fig. 15) is presented and a cluster of streamlines from the coolant hole entrance are also superimposed to illustrate the trajectory of coolant fluid particles. As the streamwise velocity of cross flow is still prominent at this unity blowing ratio, the penetration depth of the cooling jet is very small, and the jet stream bends immediately after being issued into the mainstream domain. Results from the base model and the ramp model have shown temperature contours in quite similar shape, but at different magnitude. For the ramp model, temperature level is low across the spanwise direction, in consistent with that seen in Fig. 15. For the shape diffuser mode, the temperature contours have a 'boomerang' type shape up to $x / D \approx 15$. After that, a near circular shape is developed. Not surprisingly, for console slot model, temperature contours has a 'flat' shape in the near wake region, due to the rectangular slot hole exit and this kind of shape seems very persistent up to the outlet location.

To illustrate the flow and thermal fields, Figs 19 and 20 depict velocity vectors at two streamwise positions $x / D=6,10$, superimposed with temperature contours as those shown in Fig. 18. It can be seen that a CRVP is captured by present simulation for the base (cylindrical) model, in agreement with a previous study by Kapadia et al. [5]. Furthermore, it is confirmed that the CRVP is very strong and predominant 

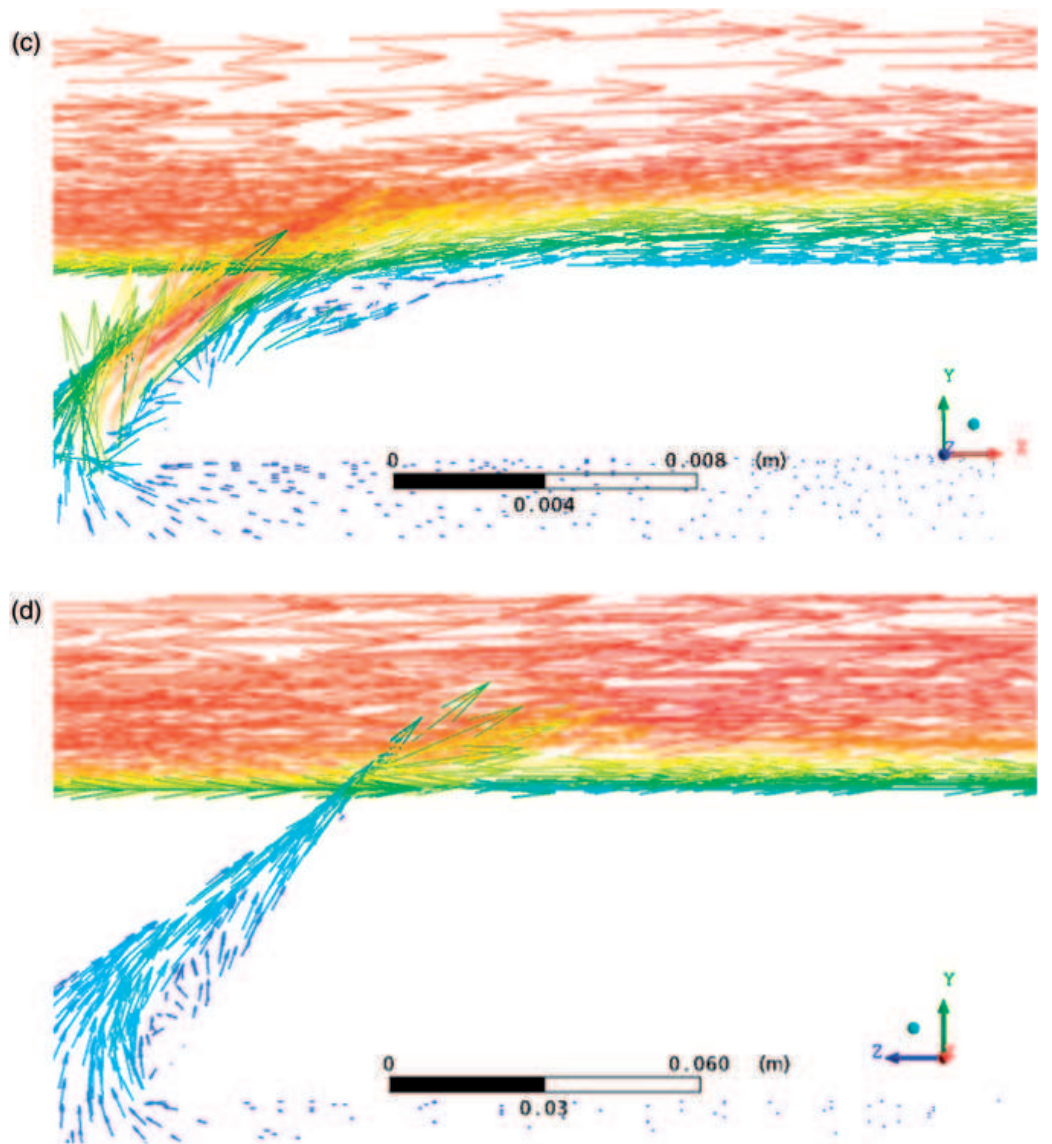

Fig. 17 Continued

up to the outlet plane. In comparison, results from the ramp model show less strong CRVP with the vortex core locations slightly drifting away from the wall surface towards the flow field. This may be due to the increase of the 'hot' mainstream flow angle (due to wedge deflection effect), thus weakening the interactions between the cooling jet flow and the mainstream flow (i.e. the interaction angle is effectively less than $90^{\circ}$ ). The shape diffuser and the console slot models have both shown much weakened vortex structure, sometimes even invisible at downstream locations, indicating that the entrainment of surrounding 'hot' gas mixture would be much less. This leads to a higher cooling effectiveness being achieved in wider area and up to longer distance from the hole exit.

\section{CONCLUSIONS}

Computational investigation of the adiabatic cooling effectiveness of a wind tunnel film=cooling test configuration has been carried out in this study. In addition to a base cylindrical (inclined tube cooling) model, three additional variants of hole shapes are considered, i.e. the ramp model with a small wedge upstream of the hole exit, the shaped diffuser model and the console slot model. All simulations apply same flow conditions for like-to-like comparisons. Validation study on the base model has found fairly good agreement with previous experimental measurement and numerical predictions at both centreline and two downstream locations for the adiabatic cooling effectiveness. For three geometry variants, comparison with available test data in the literature has been made. It was found that for the ramp model, there is limited enhancement of cooling effectiveness along the centre-line, but improvement has found in the lateral direction. For both shape diffuser model and console slot model, the adiabatic cooling effectiveness has noticeably enhanced in both streamwise and lateral directions. In particular, there is a factor of 1.5 of cooling effectiveness along the centre-line for the console model, and it increases to a factor of 2 for shape diffuser model. Further, flow structure and 


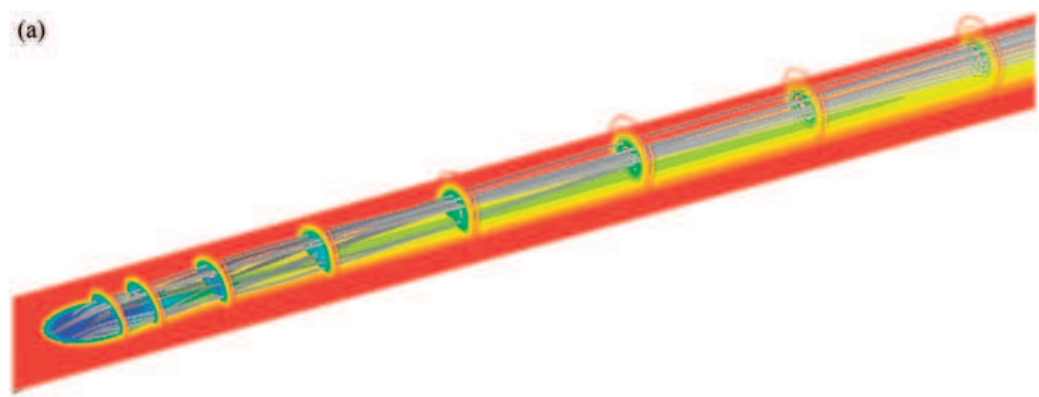

(b)
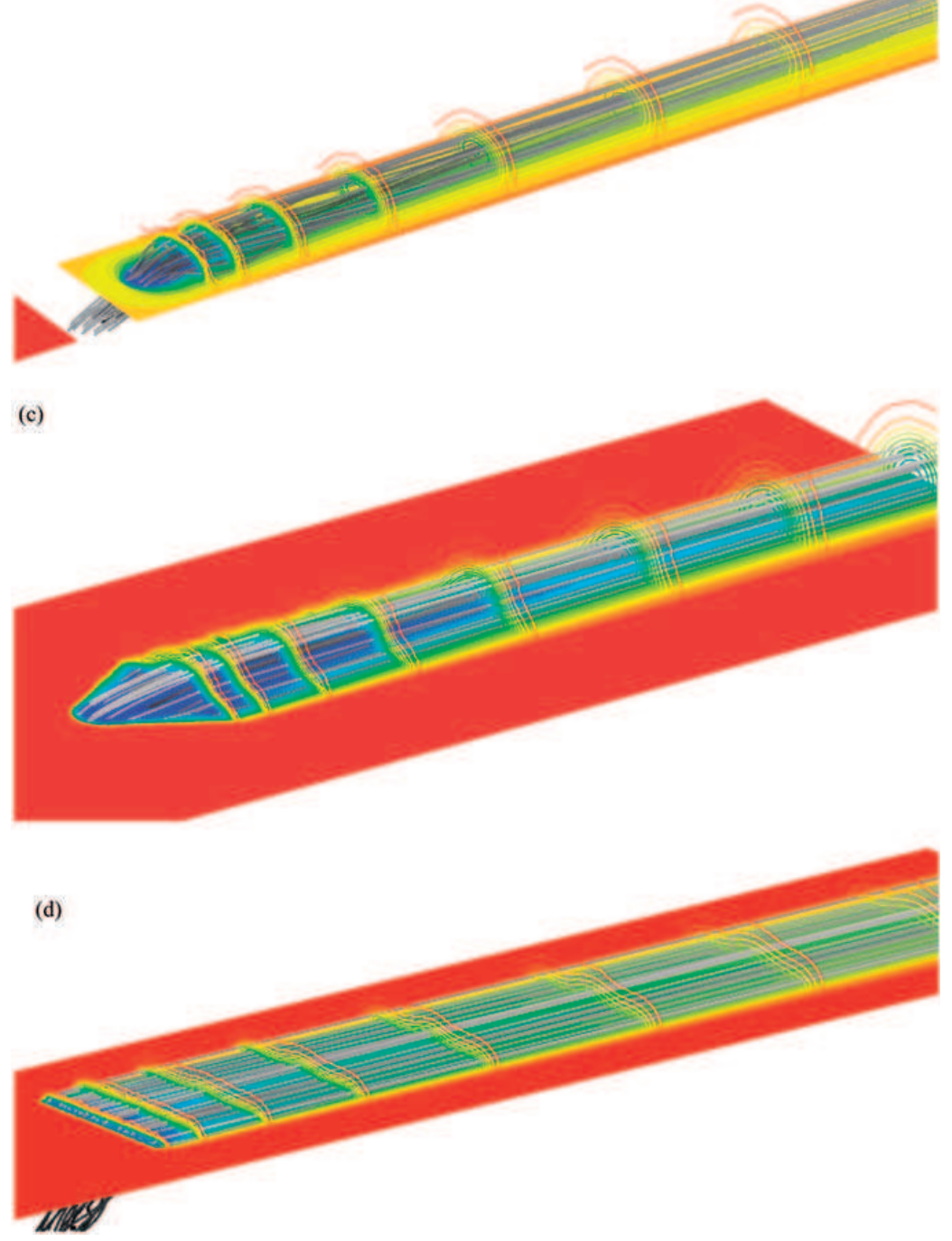

Fig. 18 Temperature distributions at cross planes of $x / D=1,3,6,10$, and 15 (for (a) base model and (b) ramp model), $x / D=0,1,3,6,10,15,20$, and 25 (for (c) shape diffuser model, and (d) console slot model) and the bottom wall of the mainstream domain. Streamlines show the evolution of coolant flow in downstream model. Temperautre contours have 16 levels from 150 to $300 \mathrm{~K}$ 
(a)

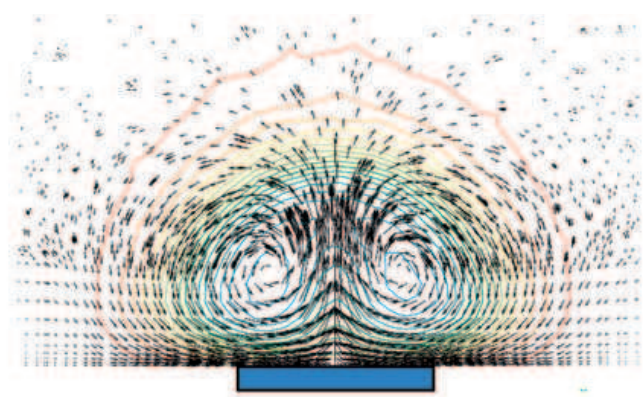

(c)

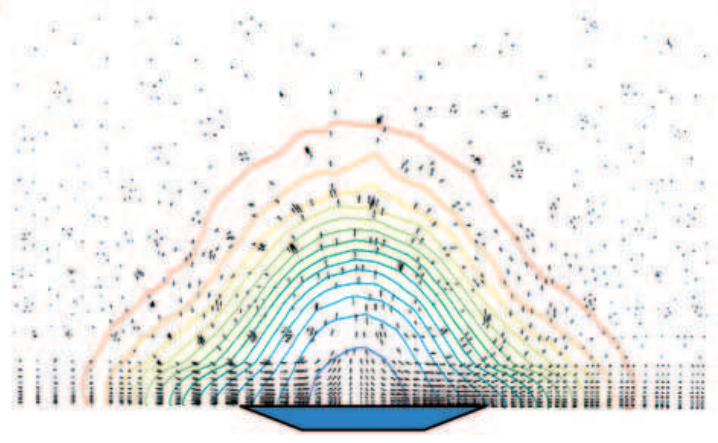

(b)

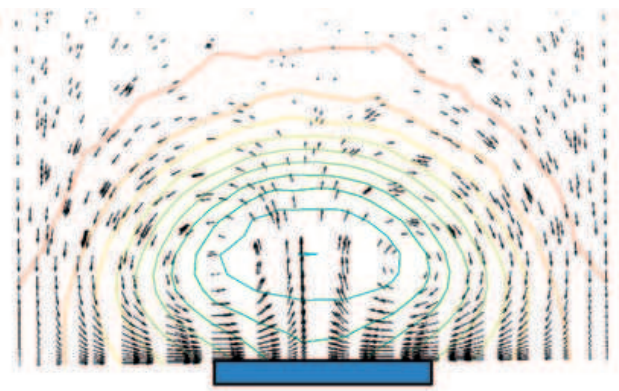

(d)

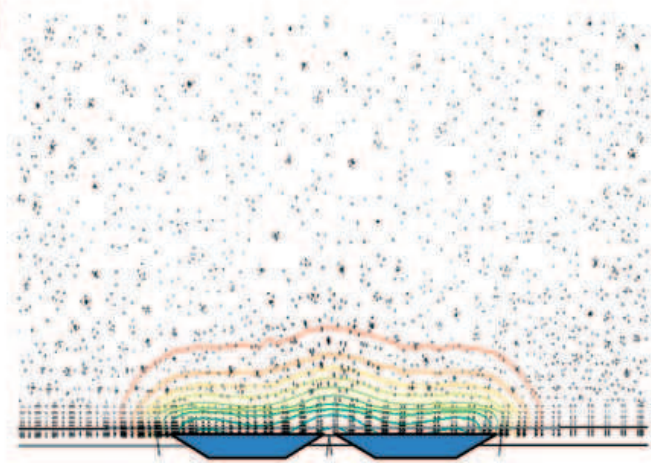

Fig. 19 Velocity vectors with superimposed temperature contours (at a range of 150-300 K with an increment of $10 \mathrm{~K}$ ) at $x / D=6$ cross plane: (a) base model, (b) ramp model, (c) shaped diffuser model, and (d) console slot model

(a)

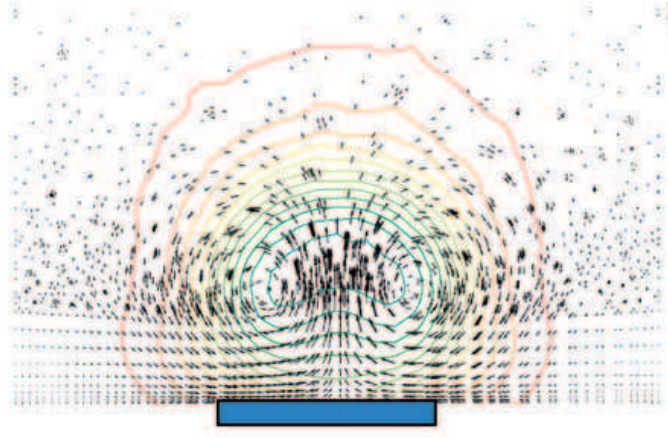

(b)

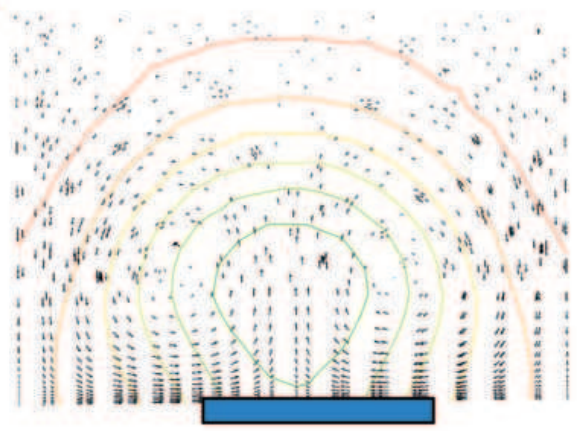

(d)

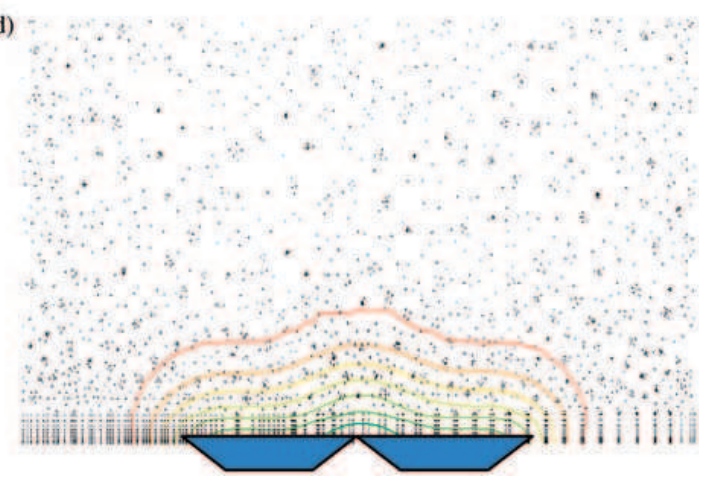

Fig. 20 Velocity vectors with superimposed temperature contours (at a range of 150-300 K with an increment of $10 \mathrm{~K}$ ) at $x / D=15$ cross plane: (a) base model, (b) ramp model, (c) shaped diffuser model, and (d) console slot model 
thermal field analysis indicated that strong CRVP observed in the base model has been weakened due to hole geometry changes in other three models. It is believed that the entrainment effect during the 'hot-cold' flow mixture process has been much weakened, leading to high level of adiabatic cooling effectiveness

(C) Authors 2011

\section{REFERENCES}

1 Cho, H. H., Rhee, D. H., and Kim, B. G. Enhancement of film cooling performance using a shaped film cooling hole with compound angle injection. JSME Int. J., Ser. B, 2001, 44(1), 99-110.

2 Teng, S. Y., Han, J. C., and Poinsatte, P. E. Effect of film-hole shape on turbine-blade film-cooling performance. J. Therm. phys. Heat Transfer, 2001, 15(3), 257-265.

3 Baheri, S., Alavi Tabrizi, S. P., and Jubran, B. A. Film cooling effectiveness from trenched shaped and compound holes. Int. J. Heat Mass Transfer, 2008, 44(8), 989-998.

4 Zhang, X. Z. and Hassan, I. Numerical investigation of heat transfer on film cooling with shaped holes. Int. J. Numer. Methods Heat Fluid Flow, 2006, 16(8), 848-869.

5 Kapadia, S., Roy S., and Heidmann, J. Detached eddy simulation of turbine blade cooling. In 36th AIAA Thermophysics Conference, AIAA-2003-3632, Orlando, Florida, 23-26 June 2003.

6 Jiang, Y., He, L., and Yu, J. Numerical investigation on heat transfer of film cooling with an upstream ramp. Proc. Chinese SEE, 2008, 28(5), 69-73.
7 Dhungel, A. Film cooling from a row of holes supplemented with anti-vortex holes. MSc Thesis, 2002 (Louisiana State University).

8 Sargison, J. E. Development of a novel film cooling hole geometry. PhD Thesis, 2001 (University of Oxford).

9 ANSYS workbench and CFX user's guide.

10 Sinha, A. K., Bogard, D. G., and Crawford, M. E. Film-cooling effectiveness downstream of a single row of holes with variable density ratio. J. Turbomach., 1991, 113, 442-449.

\section{APPENDIX}

\section{Nomenclature}

$D$ hole diameter

$h$ wedge height

$m$ blowing ratio

$\mathrm{R}_{\mathrm{e}} \quad$ Reynolds number

$S$ exit throat slot height

$T_{\text {cf }}$ eross-flow temperature

$T_{\mathrm{j}}$ coolant jet temperature

$T_{\text {aw }}$ adiabatic wall temperature

$u_{\text {cf }}$ cross flow velocity

$u_{j} \quad$ jet inlet velocity

$W$ exit slot width (or console hole pitch)

$x, y, z \quad$ Cartesian coordinates

$\alpha$ inclination angle

$\beta$ defection angle

$\Delta y^{+} \quad$ grid size in wall unit

$\eta \quad$ adiabatic cooling effectiveness

$\theta$ expansion angle

$\rho_{\text {cf }}$ cross flow density

$\rho_{\mathrm{j}} \quad$ coolant jet density 